\title{
Thermal Modeling of the Mars Reconnaissance Orbiter's Solar Panel and Instruments During Aerobraking
}

\author{
John A. Dec, Joseph F. Gasbarre, and Ruth M. Amundsen \\ NASA Langley Research Center
}

\begin{abstract}
The Mars Reconnaissance Orbiter (MRO) launched on August 12, 2005 and started aerobraking at Mars in March 2006. During the spacecraft's design phase, thermal models of the solar panels and instruments were developed to determine which components would be the most limiting thermally during aerobraking. Having determined the most limiting components, thermal limits in terms of heat rate were established. Advanced thermal modeling techniques were developed utilizing Thermal Desktop and Patran Thermal. Heat transfer coefficients were calculated using a Direct Simulation Monte Carlo technique. Analysis established that the solar panels were the most limiting components during the aerobraking phase of the mission.
\end{abstract}

\section{INTRODUCTION}

MRO launched on August 12, 2005 and started aerobraking at Mars in March 2006. Aerobraking is a propellant saving technique by which a spacecraft makes successive passes into a planet's atmosphere. Atmospheric drag reduces the spacecraft's periapsis velocity thereby reducing the apoapsis altitude after each pass through the atmosphere. The higher the drag, the higher the resulting $\Delta \mathrm{V}$ for a given drag pass. The spacecraft passes through the upper atmosphere at hypersonic speeds and as a result is subjected to an aerodynamic heat load. This aerodynamic heat load causes the temperature of the spacecraft and its components to increase during the drag pass. To make an obvious observation, the higher the heat load, the higher the resulting temperature. The atmospheric drag and the aerodynamic heating are both functions of the atmospheric density and spacecraft velocity. This fact results in one of the fundamental trades in performing an aerobraking maneuver: $\Delta \mathrm{V}$ versus heating. As the spacecraft passes deeper into the atmosphere, the density increases, which results in larger drag and a larger $\Delta \mathrm{V}$. However, as the density increases, so does the aerodynamic heating, which results in higher spacecraft temperatures.

One challenge associated with aerobraking is to predict temperatures during a drag pass and also establish thermal limits in terms of heat rate which the spacecraft can not exceed during flight. After adding sufficient margin, a heat rate corridor is established and the spacecraft's trajectory is then designed so that it flies within the upper and lower bounds of the corridor. To accomplish the task of calculating temperatures and establishing the thermal limits, thermal models are developed to represent the spacecraft and its main components such as the solar panels and instruments.

\section{ANALYSIS OBJECTIVES}

Prior to aerobraking operations, the goal of the thermal analysis of the solar array and instruments was to generate thermal limit lines, assess mission risk during aerobraking, and identify the most limiting components (from a thermal standpoint). The analysis performed by NASA Langley Research Center was primarily done for validation and verification, while the responsibility for the design was with the project contractors. A thermal limit line, which will be discussed in more detail later, is a plot of maximum heat flux as a function of the mission timeline. The mission timeline can be tracked either by orbit pass number, orbit period, or drag pass duration. This line defines the maximum heat flux a spacecraft component may encounter without exceeding its flight allowable temperature, and upon examining the thermal limit line for each component, the one which displays the lowest allowable heat flux for its specific flight temperature limit, is the most limiting. The component with the highest risk associated with it is the one that has the least amount of margin with respect to the ERD, or the environmental requirements document. For MRO aerobraking, the solar array was most limiting and the one with the most risk. The ERD defined the maximum allowable heat flux the spacecraft would need to be able to safely fly though without exceeding any temperature limits. Thus, the thermal limit lines for the solar array and instruments all needed to be greater than the ERD. For MRO, the flight corridor through the Martian atmosphere was defined based on a not-to-exceed heat flux as well as what local mean solar time was required for the science orbit. The mission environment turned out to be $200 \%$ less than the required maximum allowable environment, i.e., the ERD line.

During aerobraking operations the goal of the analysis was to predict peak temperatures of the solar array thermal sensors for each upcoming orbit pass and 
correlate them using flight data ${ }^{1}$. The analysis was also used to predict the over all maximum temperature in the array in the event it was not located near any of the four sensors. The prediction of the maximum temperature, as well as the prediction of the sensor temperatures was used to develop a response-surface model of the solar array. The response-surface model was embedded in a Monte Carlo simulation from which a 3-sigma temperature bound was estimated as well as an estimation of the probability of exceeding the solar array's flight allowable temperature. The development and results of this probabilistic analysis are described in another work and will not be described here ${ }^{2}$.

To accomplish the objectives stated above, a detailed thermal analysis of the MRO solar array and instruments was required that made use of several analysis tools each with specific functions. The trick was to assemble all the parts in a logical and efficient way so as not to be overly cumbersome to the analyst. The three main tools used to perform the analysis were Thermal Desktop ${ }^{3}$, MSC Patran Thermal ${ }^{4}$, and a direct simulation MonteCarlo (DSMC) code called DAC.

\section{AERODYNAMIC HEATING}

The DAC and the free molecular version DAC Free were used to calculate the incident aerodynamic heat transfer coefficients. Calculations were performed over a range of atmospheric densities, for several different spacecraft pitch, and yaw angles. The analysis presented in this paper is for the nominal trim attitude which was $0^{\circ}$ pitch and yaw. The DAC model included the spacecraft bus, both solar arrays, and all the scientific instruments. The DAC model used for the MRO analysis where the arrow shows the direction of flight is shown in Figure $1^{5}$. An example of the heat transfer coefficient distribution for a density of $32 \mathrm{~kg} / \mathrm{km}$ is shown in Figure $2^{3}$.

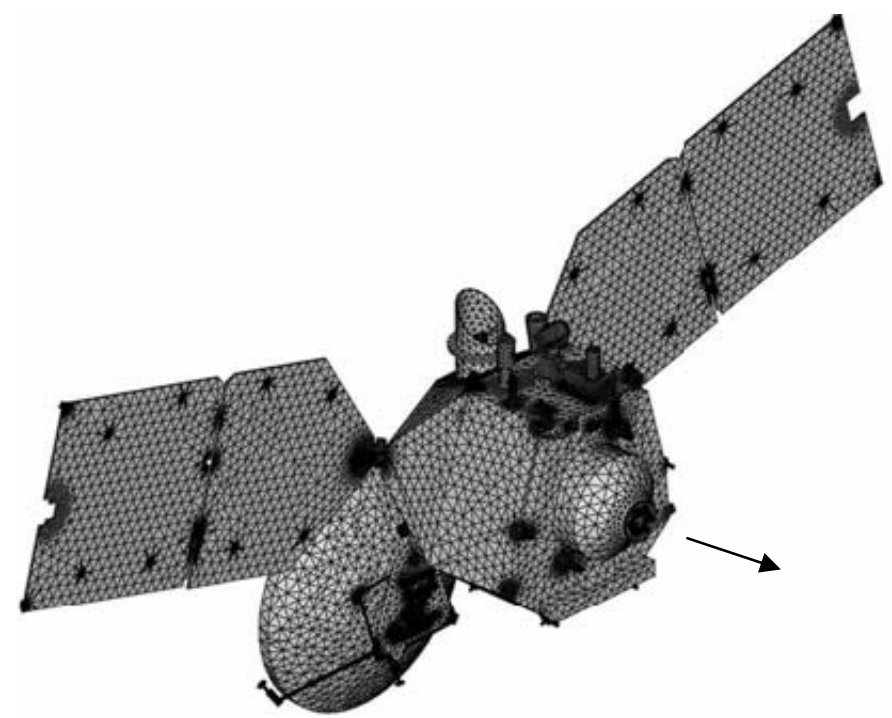

Figure 1. DAC computational geometry

The locations of the thermal sensors on the solar array are also shown in Figure 2. Largely due to the lessons learned from the Mars Odyssey aerobraking experience, for the design of the MRO solar arrays, every effort was made to position the thermal sensors in the areas of the

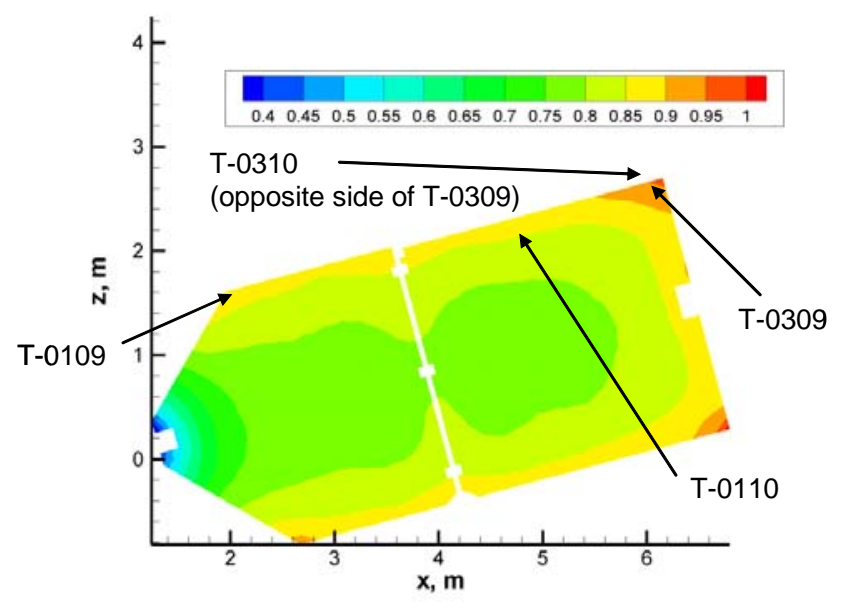

Figure 2. MRO dimensionless heat transfer coefficient distribution with sensor locations for a density of $32 \mathrm{~kg} / \mathrm{km} 3$

array that would show the highest temperatures during aerobraking ${ }^{6,7}$. Plots like Figure 2 as well as plots of the temperature distribution served as a guide for designers to correctly position the sensors. Heat transfer coefficients were calculated for every component of interest. For the thermal analysis, the solar arrays, as well as the instruments HiRISE, CTX, MARCI, SHARAD, MCS, and CRISM were the components that were of interest and needed to be evaluated for aerobraking. A close up view of the DAC model of the nadir instrument deck for a density of $32 \mathrm{~kg} / \mathrm{km}^{3}$ is shown in Figure 3 with the same scale as that in Figure 2. Note that heat

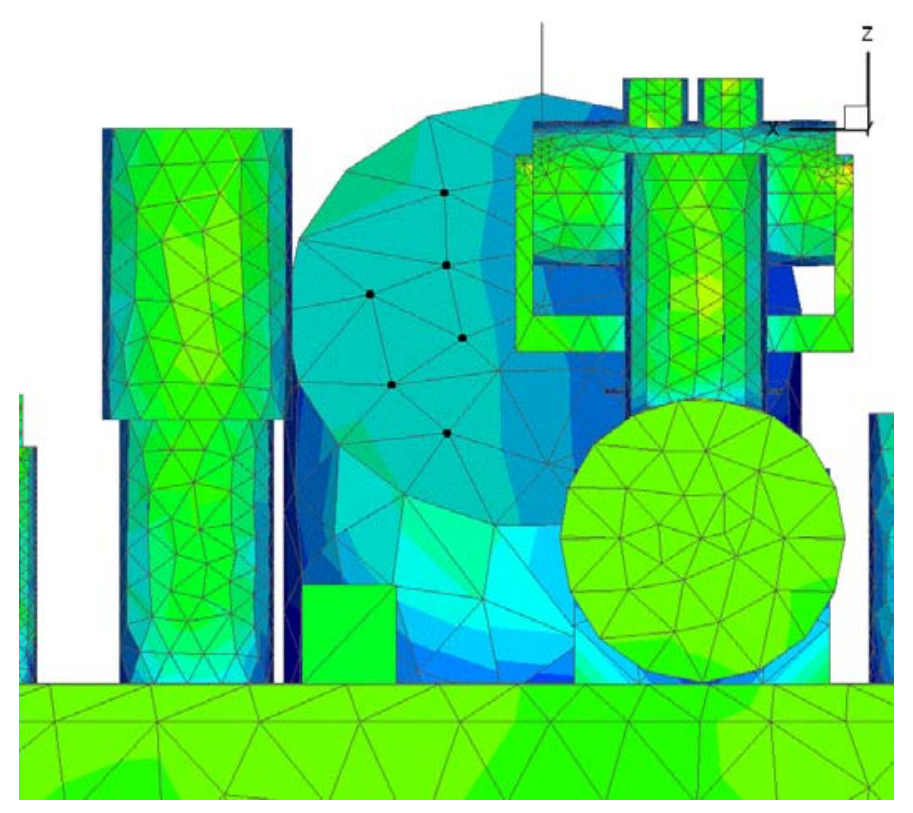

Figure 3. Nadir instrument deck heat transfer coefficients for a density of $32 \mathrm{~kg} / \mathrm{km} 3$ 
transfer coefficients for the instruments are more benign than that of the solar array. The instrument deck experienced overall lower heat transfer rates due to the presence of a bow shock that formed on the front end of the vehicle which provided some relief for the instrument deck.

Once the heat transfer coefficients $\left(\mathrm{C}_{\mathrm{H}}\right)$ were calculated as a function of density and spacecraft orientation, the aerodynamic heat flux incident on each component could be calculated. The heat flux, Q, was calculated using equation 1 , where $\rho$ is the atmospheric density and $v$ is the spacecraft velocity. The DSMC calculations also provided reflected heat transfer coefficients. Reflected heating occurs due to the elevated temperatures of the solar array and instrument surfaces when gas particles collide with the surface and rebound with more energy than they came in with. The total $\mathrm{C}_{H}$ is simply the reflected $\mathrm{C}_{\mathrm{H}}$ reflected subtracted from the incident $\mathrm{C}_{\mathrm{H} \text { incident }}$.

$$
Q=\frac{1}{2} \cdot \rho \cdot V^{3} \cdot C_{H_{\text {incident }}}
$$

The spacecraft velocity and density as a function of time were provided by the flight mechanics calculations for each orbit drag pass. From this trajectory, the heating as a function of time was calculated.

\section{ORBITAL HEATING AND VIEW FACTORS}

Cullimore and Ring's Thermal Desktop software was used to calculate the solar and planetary heat rates as well as the view factors to space for the MRO spacecraft. The Thermal Desktop model was developed manually from the geometry and drawings of the MRO spacecraft. The Thermal Desktop model is shown in figure 4. The spacecraft bus is included in this model in order to have the shadowing effects and reflections to the solar arrays correct. In the Thermal

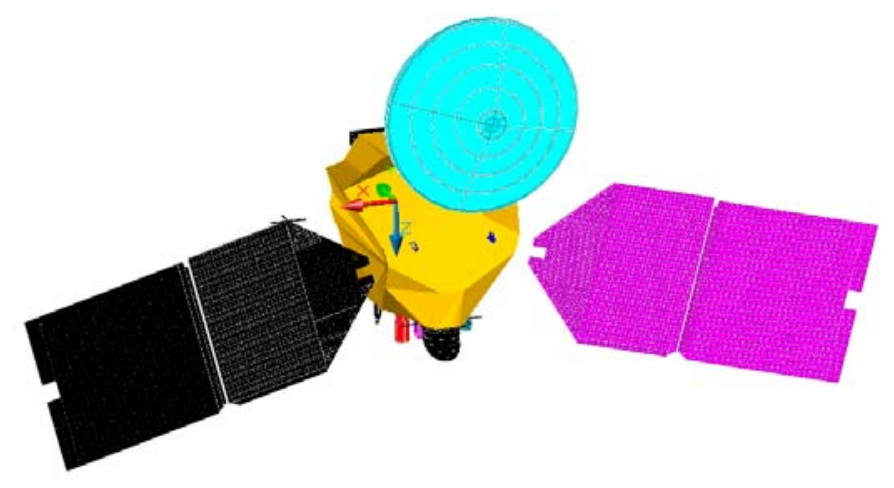

Figure 4. Thermal Desktop model of the spacecraft, solar arrays and instruments
Desktop model, only exterior optical properties are included. The output from this model is a set of solar and planetary heating maps for a number of positions around the entire orbit for each of ten different orbits. The view factors to space were also calculated for the same orbit positions. The Thermal Desktop model has 725 surfaces in the entire model, with 4488 nodes on each solar array. The run of a heat rate case for a single orbit in Thermal Desktop takes approximately 100 minutes to complete on an Intel $3.20 \mathrm{GHz}$ Dual Xeon processor PC. In much the same way as the database of heat transfer coefficients was created, a database of solar and planetary heat rates as well as view factors were generated. The ten orbits chosen for the database spanned the aerobraking phase of the mission from start to finish. Within each orbit, 19 orbit positions were used. These positions essentially gave the solar and planetary heat flux and the view factor data as a function of time in the orbit.

The orbital heating, view factors, the DSMC $\mathrm{C}_{\mathrm{H}}$ databases, and the flight trajectory represented the major environmental boundary conditions to the Patran thermal model. Applying these boundary conditions is the subject of a later section.

\section{FINITE ELEMENT THERMAL MODEL}

MSC Patran Thermal was used as the thermal modeling and temperature solver to calculate the 3-D temperature distribution of the solar arrays and instruments. The Patran Thermal model for the MRO solar array is shown in figure 5. The model is of only a single solar array since the nominal attitude was $0^{\circ}$ pitch and yaw which was assumed to produce symmetric temperature distributions in the arrays. The model contains 88,650 nodes and 73,300 elements and consists of five layers, two for the composite facesheets, one for a central aluminum honeycomb core, one for the Kapton sheet, and one for the solar cell layer. Masses of cabling, solder, and adhesive for the solar cells are smeared evenly in the solar cell layer. The as-built mass of the

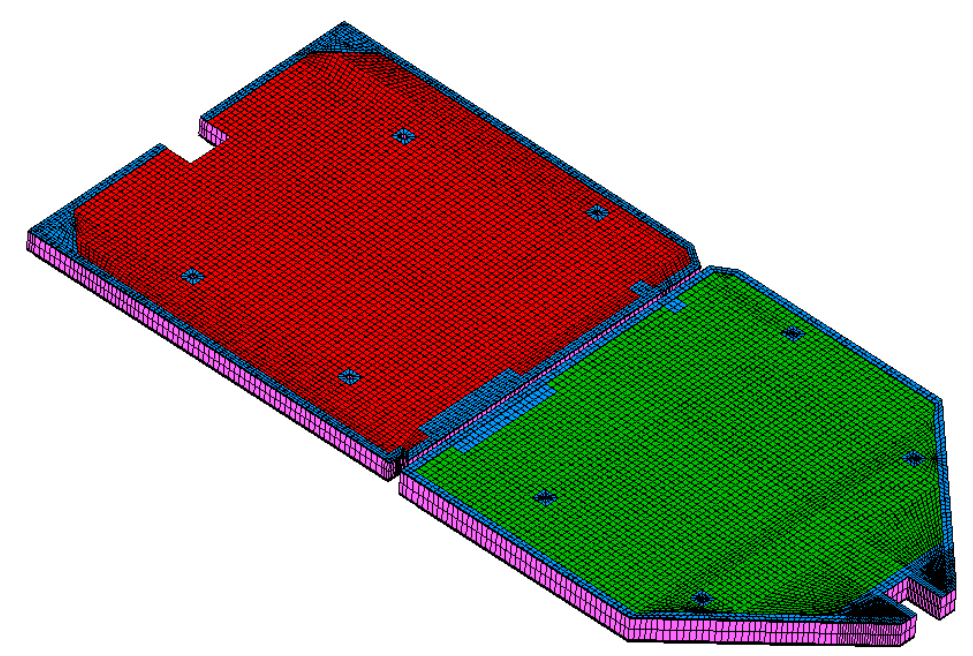

Figure 5. Patran Thermal model of MRO solar array 
MRO solar array is $48.3 \mathrm{~kg}$, while the Patran model mass $47.5 \mathrm{~kg}$. The model is thus slightly conservative in terms of mass, being $1.6 \%$ light. Incorporated in the model are the differing thickness of facesheets and core between the inboard and outboard sections, differing densities and thickness at the hard points and hinge connections, and a Kapton layer around the outer circumference of each section, which adds a small amount of mass, and is intended to protect the edges of the array from the higher heating rates.

Similar Patran thermal models were developed for the instruments. The Patran thermal model of the HiRISE telescope is shown in figure 6 . Even though the geometry of the instruments are different than that of the solar array, they still follow the same procedure for incorporating the boundary conditions which demonstrates the utility and generality of this analysis methodology.
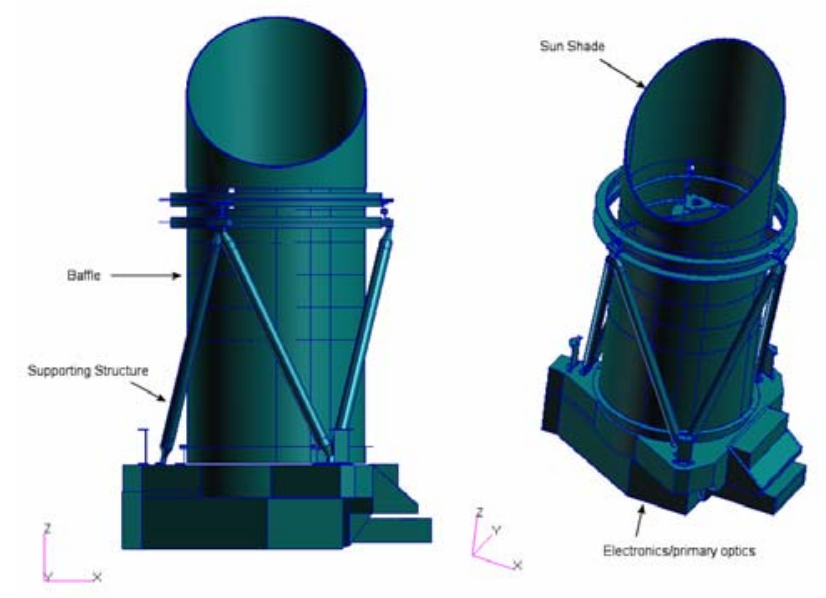

Figure 6. Patran thermal model of the HiRISE instrument

\section{ASSEMBLING THE PIECES}

As previously mentioned, the boundary condition inputs were calculated using other software tools and were provided in a tabular text file format. For the DSMC $\mathrm{C}_{\mathrm{H}}$ data, each file represented a different atmospheric density and spacecraft attitude. Again, since the nominal attitude was $0^{\circ}$ pitch and yaw, only one set of $\mathrm{C}_{\mathrm{H}}$ as a function of density was needed. The raw data file contained the DAC model's nodal $x, y, z$ coordinate

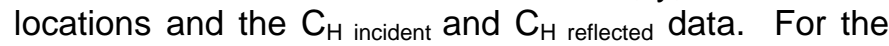
view factors and orbital heating rates, each file represented a different time point in the orbit. These Thermal Desktop output files contained the model's nodal $x, y, z$ coordinate locations and the value of the variable of interest. Each software tool had its own unique computational mesh, but, all three models were constructed using the same geometric coordinate system and system of units.
In order to use the boundary condition data in this text file form, the data from DAC and Thermal Desktop had to be mapped to the Patran mesh. The desired format was to have a two-column table in a text file containing the Patran model's nodal reference number along with the data of interest. The mapping process was accomplished by using a Tecplot macro which utilized Tecplot's built-in inverse distance interpolation routine. The only input required by the macro, in addition to the database files, was a text file containing the Patran node numbers and their $x, y, z$ coordinate locations. The output of the macro was a set of files which were in the desired format, Patran node number and data value. Having the data already mapped to the Patran mesh in this way eliminated the need to perform spatial interpolations while the analysis was running, and represented a significant improvement over previous aerobraking thermal analyses ${ }^{6,7}$. The Program to Optimize Simulated Trajectories (POST) was used to produce trajectory input files with density and velocity given as a function of time; the only interpolations needed during the Patran run were in time in the case of the orbital heating and in density for the $\mathrm{C}_{\mathrm{H}}$. To use this data within the Patran model, several customizations using Patran's ULIB user FORTRAN were made. Patran's ULIB library is a FORTRAN library that is linked with the Patran analysis executable at run time. This library allows the user to develop subroutines that interface directly with the Patran variables such as node number, nodal coordinates, analysis time step, nodal temperature, material properties, etc. Reference 4 contains a complete list of variables that may be accessed by the user. In the case of the MRO thermal analysis, the aerodynamic heating, orbital heating, and radiation to space boundary conditions were applied using the ULIB functionality.

The first customization was to read in the mapped database files and store them in arrays. These data arrays were then accessed as necessary during the transient thermal run by the user developed linear interpolation routine within the ULIB library. The next customization was to apply the boundary conditions by assigning nodal values for heat flux. At each time step, the interpolation subroutine interpolated the orbital heating and view factor database to get the nodal values for the orbital heating and view factors taking the time and node number as input. The $\mathrm{C}_{\mathrm{H}}$ total for the current time step was calculated from the interpolated values of

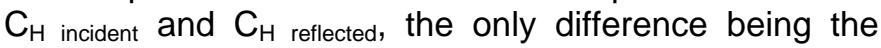
interpolation routine took density and node number as input. Next, using the calculated $\mathrm{C}_{\mathrm{H}}$ total, the aerodynamic heat flux could be calculated for the current time step using equation 1 and applied to the current node. The incident solar heating value obtained from the interpolation was simply added to the value of the aerodynamic heat flux. For the radiation to space, the radiative heat flux was calculated within the user FORTRAN, thus bypassing the standard radiation boundary condition within Patran. The radiative heat flux was calculated using equation 2 at each time step for the current node. In equation 2 , the 


$$
Q_{\text {rad }}=\varepsilon \cdot f_{i j} \cdot \sigma \cdot\left(T_{n}^{4}-T_{\sin k}^{4}\right)
$$

emissivity, $\varepsilon$, for each material that had a radiation to space boundary condition applied, was read from an external file and stored in an array. The view factor, $f_{i j}$ was obtained from the interpolation of the Thermal Desktop data, as previously discussed. $\mathrm{T}_{\mathrm{n}}$ and $\mathrm{T}_{\text {sink }}$ are the current nodal temperature and radiation sink temperatures, respectively. The radiative heat flux given by equation 2 was then subtracted from the total of the aerodynamic heating and solar heating. The total heat flux applied to the current node at the current time step was then given by equation 3 .

$$
Q_{\text {total }}=Q_{\text {aero }}+Q_{\text {solar }}-Q_{\text {rad }}
$$

There are two distinct advantages in applying the boundary conditions in this manner. The first is that it allows the Patran transient to be continuous throughout an entire orbit which allows inclusion of orbital events such as the spacecraft slewing into its aerobraking attitude, and the abrupt change in solar heating due to the spacecraft passing through the solar occultation region. Then the preconditioning of the solar arrays and instruments prior to the start of the drag pass can be simulated. Including the orbital events and simulating the orbital transient prior to the start of the drag pass produces accurate starting temperatures and accurate temperature gradients. Although accurate prediction of the atmospheric density is by far the most important, having accurate starting temperatures and gradients were also shown to be a major driver in predicting the peak temperatures during a drag pass ${ }^{1,6}$. The second advantage is in the computational run time and the time it would take to set up and run a full orbit transient using the standard radiation boundary condition within the main Patran software. The standard radiation to space boundary condition does not allow the user to easily incorporate view factors that are a function of time. To do so one would have to set up several shorter transients, (between the orbit points from Thermal Desktop), and run the orbit transient piece-by-piece, copying the final temperature results to the next succeeding transient to use as starting temperatures. Calculating the radiative heat flux as a negative heat input alleviates this restriction since in the user FORTRAN the view factor is free to be a function of time. The aerobraking thermal analysis sequence is summarized in figure 7 . The lighter shaded blue boxes represent the input and pre-processing, the darker blue the Patran transient run, and the orange the output from Patran.

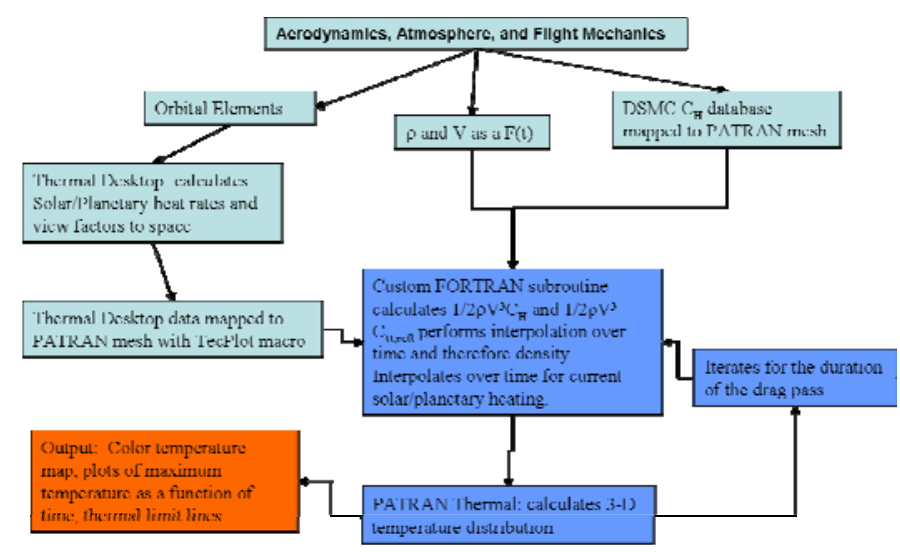

Figure 7 Analysis sequence for MRO aerobraking

\section{ANALYSIS RESULTS}

With the analysis process defined, the task was then to establish the thermal limits for the solar arrays and instruments. As mentioned previously, the thermal limit lines serve to define the maximum heat flux any component may encounter without exceeding its flight allowable temperature. The thermal limits are a function of mostly the peak heat flux encountered and the length of the drag pass, i.e. the total heat load. The thermal limits also depend to a lesser extent on the mass of the particular component and its maximum allowable temperature.

Before discussing the thermal limit results for each individual instrument and the solar array, a more detailed description of how thermal limit lines are generated is warranted. The thermal limit line for the mission is calculated by running the PATRAN model at several "points" during the aerobraking phase. These "points" are really just a snapshot at various times during the aerobraking phase and are typically identified either by drag pass duration, the orbit period, or revolution number. For each orbit and, thus, drag pass being examined, the PATRAN thermal model is run four times. Each of the four runs has a slightly different trajectory that reaches a different peak heat flux as shown in figure 8. 


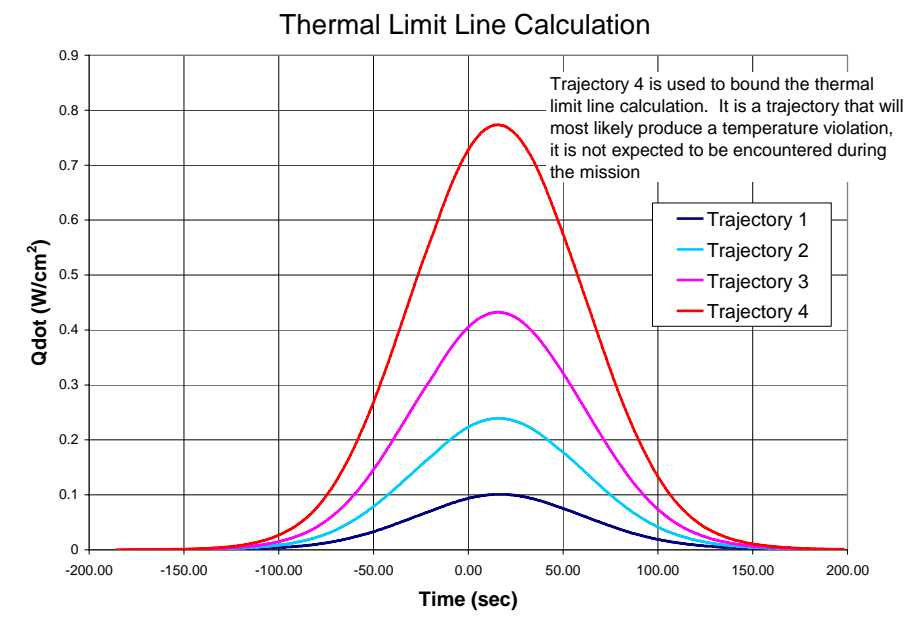

Figure 8. Trajectories used for limit line generation

The trajectories 1 and 2 from figure 8 represent the lower and upper bounds of the flight corridor. Trajectories 3 and 4 represent trajectories that would result in elevated temperatures. Trajectory 4 was selected to ensure the temperature limits would be exceeded. The maximum temperatures for each component's critical material for each run are recorded and a cubic curve fit is developed between the four points, with temperature as the independent variable and heat flux as the dependent variable. Using the cubic curve fit, the critical material's maximum single use temperature (or agreed upon flight limit) is input and the output is the heat flux that will produce that temperature.

The results for HiRISE show that the instrument will not exceed its thermal limit at any point in the aerobraking phase of the mission. The temperature distribution on HiRISE for an 1150 second drag pass with a peak heat flux of $0.80 \mathrm{~W} / \mathrm{cm}^{2}$ is shown in figure 9 .
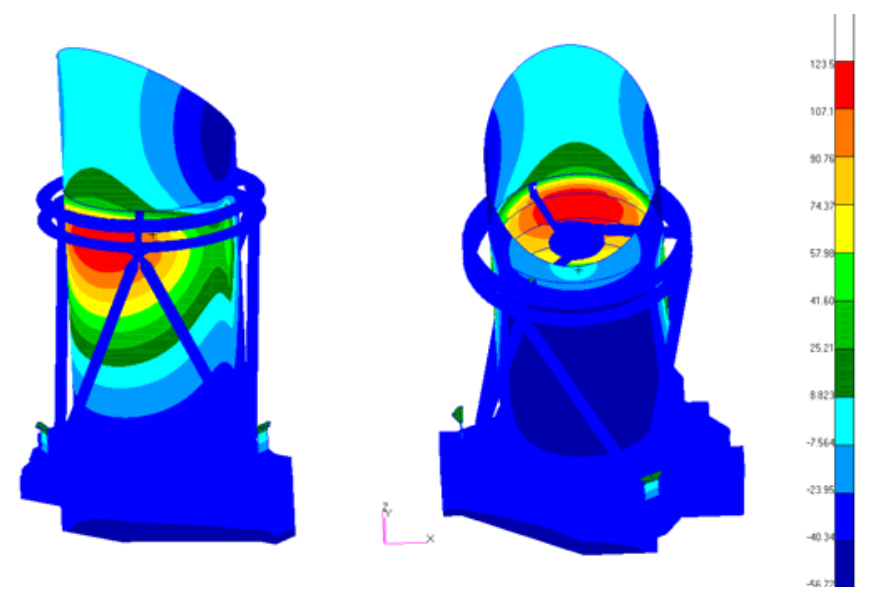

Figure 9. HiRISE temperature distribution at the time of peak temperature, 1150 second drag pass duration, $0.80 \mathrm{~W} / \mathrm{cm}^{2}$ peak heat flux
A hot spot appears in the baffle section just below the baffle-sun shade interface. For conservatism, in addition to the heating calculated using equation 1 and the calculated $\mathrm{C}_{\mathrm{H}}$ for HiRISE, an additional heat flux was applied to the inner surface of the baffle to account for the possibility of flow making its way down the baffle and interacting with it. Even with this very conservative approach, the temperature limit of the baffle and sunshade of $145^{\circ} \mathrm{C}$ was never exceeded during any of the analysis runs. Temperature distributions similar to figure 9 were calculated for each of the other five instruments, where each is able to identify hot spots for its instrument. Following the above procedure for constructing a thermal limit line, the limit line for HiRISE was constructed and compared to the ERD limit. The limit lines for the six instruments are plotted in figure 10 along with the ERD limit line. Each instrument had one specific component that was the most limiting thermally. For HiRISE it was the telescope baffle which was made from M55J graphite epoxy. For CTX, it was the MLI which surrounded the instrument. For CRISM and $\mathrm{MARCI}$, it was their radiators. CRISM had a very thin K12D2U graphite epoxy radiator which was directly in the flow during the drag pass. MARCl had a small aluminum radiator which was shaped like a taco shell. SHARAD's most limiting components were the fiberglass epoxy antenna tubes. Finally, for MCS, the aluminum camera housing was the most limiting.

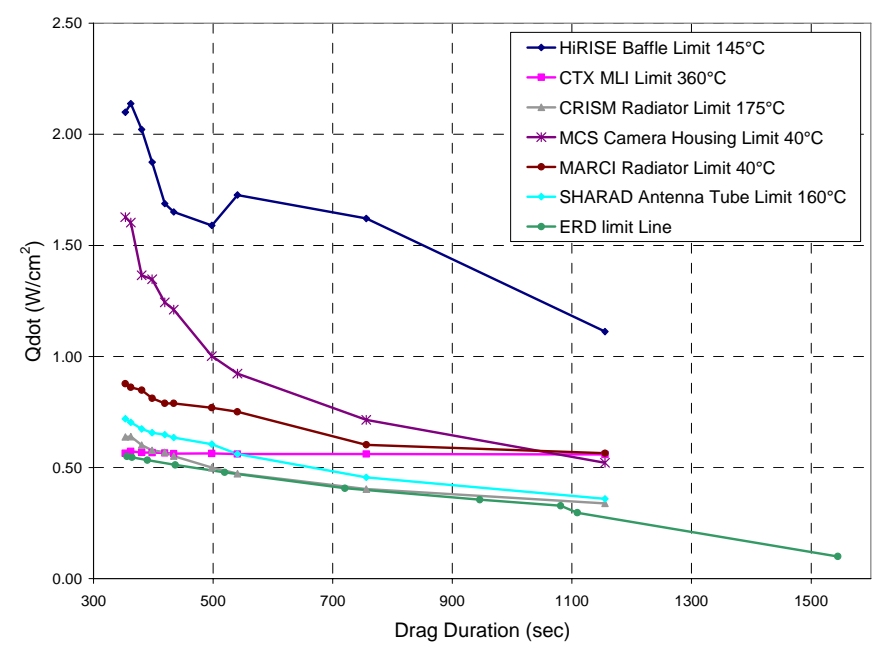

Figure 10. Instrument thermal limit lines

HiRISE had the largest amount of margin between its thermal limit and the ERD. Compared to the ERD, at its worst point in the mission there is still $217 \%$ margin between the capability of the HiRISE baffle and the ERD limit. The results presented in Figure 10 indicate that CRISM and in the beginning of the mission, CTX have the least amount of margin with respect to the ERD. However, CTX had essentially an extra $40^{\circ}$ margin in the MLI since the manufacturers stated multi-use temperature limit was $400^{\circ} \mathrm{C}$. The flight allowable was 
set to $360^{\circ} \mathrm{C}$ so that the $400^{\circ}$ would have a very low probability of being reached. CRISM on the other hand had to have its flight allowable temperature raised from the original $45^{\circ} \mathrm{C}$ to the current $175^{\circ}$ in order to just satisfy the ERD. The CRISM radiator was post-cured to $232^{\circ} \mathrm{C}$, so raising the flight allowable did not pose a problem in fact the radiator still had an additional $57^{\circ} \mathrm{C}$ margin. SHARAD was also close to the ERD, but the fiberglass epoxy limit of $160^{\circ} \mathrm{C}$ was conservatively set and since it exceeded the ERD there was no reason to raise the limit any further.

Compared to the instruments, the solar array exhibited the least amount of margin with respect to the ERD. Solar arrays have been the most limiting components for all previous aerobraking missions to date, and MRO is no exception ${ }^{6,7,8,9}$. An example of the temperature distribution for the MRO solar array is shown in figure 11 , with the through the thickness scale expanded for clarity.

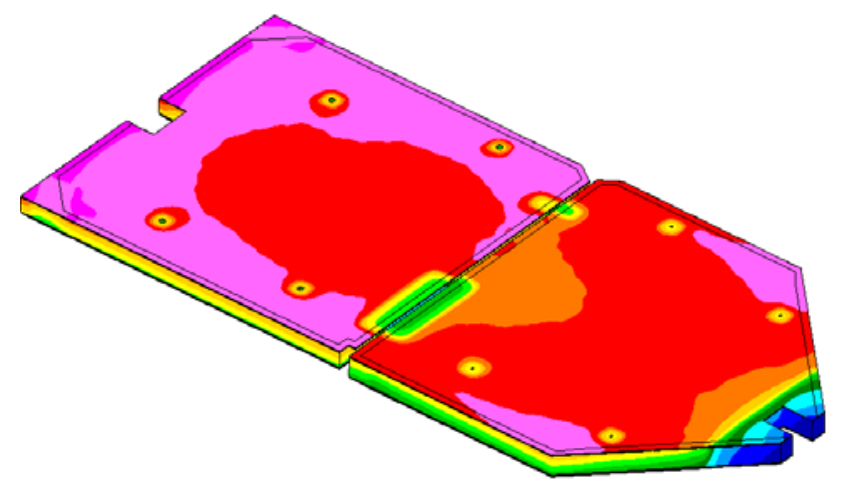

Figure 11. Temperature distribution for limit line curve $3\left({ }^{\circ} \mathrm{C}\right)$

This temperature distribution was generated using curve three from the set of four limit line trajectories. The thermal limit line of the solar array is compared to the ERD in figure 12. Note the difference in y-axis scale between figure 10 and 12 . The only instrument as close to the ERD limit line as the solar array was CRISM. CRISM had more margin to work with than did the solar array which makes the solar array the overall most limiting. As stated above,

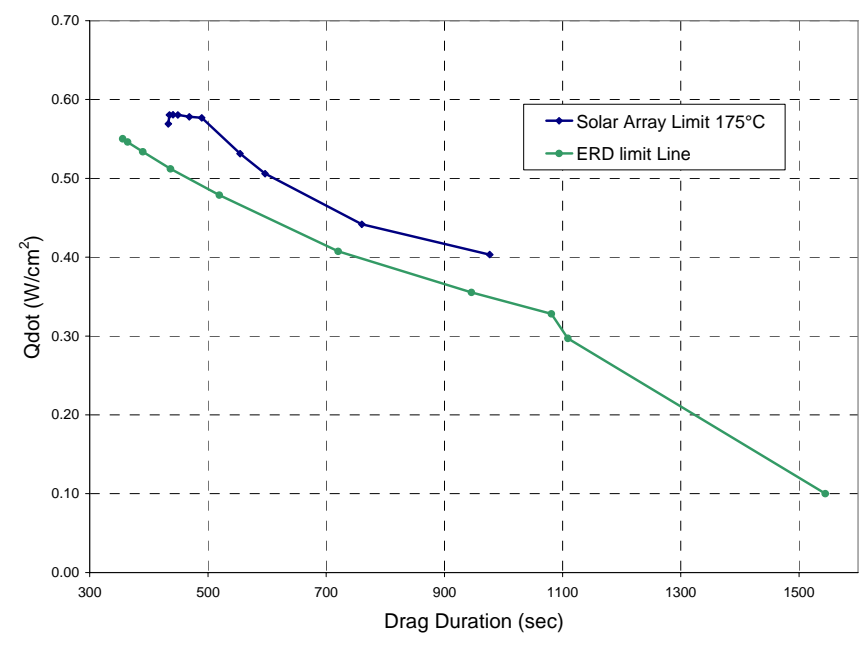

Figure 12. Thermal limit line for the MRO solar array

the CRISM radiator had been post cured to $232^{\circ} \mathrm{C}$ which gave it additional capability compared to its $175^{\circ}$ flight allowable. The limiting material in the solar array was the M55J graphite facesheet which was exposed to the flow during the drag pass. This material had only been qualified to $195^{\circ} \mathrm{C}$ which did not give it much additional margin with respect to its flight allowable of $175^{\circ} \mathrm{C}$. It was for this reason that the solar array became the most limiting for the spacecraft during the main phase of aerobraking. The limit line for the solar array starting later with respect to the drag pass duration is also shown in figure 12. This result occurred since the shorter drag passes occurred during aerobraking "walkin", where the spacecraft would gradually insert itself into the aerobraking corridor, and it was deemed that walk-in would not pose a thermal problem for the solar array. It was unknown at the time of this analysis whether or not walk-in would pose a problem for the instruments, so they were analyzed during main phase aerobraking and for walk-in.

\section{CONCLUSION}

An efficient and comprehensive methodology for performing thermal analysis during aerobraking has been presented. This methodology increases the level of fidelity early on in the design phase and is a great asset during operations. The methodology is general so that any number of spacecraft components may be analyzed. Much insight was gained by examining the temperature distributions and limit lines for the instruments and solar array. Based on this analysis, the flight allowable limit for CRISM was increased. The high fidelity modeling presented here was instrumental in being able to correlate the thermal model to flight data and was essential in being able to accurately represent the solar array with a response surface model during operations. 


\section{ACKNOWLEDGMENTS}

The support of the entire Mars Reconnaissance Orbiter Navigation team is gratefully acknowledged; in particular the support of Jill Prince, Scott Striepe, Jeremy Shidner, Richard Wilmoth and Derek Liechty has been invaluable. Also, the support of Christian May and Neil Tice at Lockheed Martin is gratefully acknowledged.

\section{ACRONYMS}

CRISM Compact Reconnaissance Imaging Spectrometer for Mars

CTX Context Camera

DSMC Direct Simulation Monte-Carlo

ERD Environmental Requirements Document

HiRISE High Resoultion Imaging Science Experiment

MARCI Mars Color Imager

MCS Mars Climate Sounder

MGS Mars Global Surveyor

MLI Multi-layer Insulation

MRO Mars Reconnaissance Orbiter

POST Program to Optimize Simulated Trajectories

SHRADShallow Subsurface Radar

Qdot Aerodynamic Heat Rate $\left(\mathrm{W} / \mathrm{cm}^{2}\right)$

\section{REFERENCES}

1. Amundsen, R. M., Dec, J. A., Gasbarre, J. F., "Thermal Model Correlation of the Mars Reconnaissance Orbiter," 07ICES-17, 37th International Conference On Environmental Systems, Chicago, Illinois, July 2007.

2. Dec, J. A., "Probabilistic Thermal Analysis of the Mars Reconnaissance Obiter's Solar Array During Aerobraking," AIAA-2007-1214, AIAA 45th Aerospace Sciences Meeting and Exhibit, Reno, NV, January 8-11, 2007.

3. Thermal Desktop user manual, Cullimore and Ring Technologies, Inc., Version 4.7, October 2004.

4. MSC/PATRAN User Manual, MacNeal-Schwendler Corporation, Version 2005, December 2004.

5. Leichty, D. S., "Aeroheating Analysis for the Mars Reconnaissance Orbiter with Comparison to Flight Data," AIAA 2006-3890, 36th AIAA Fluid Dynamics Conference and Exhibit, San Francisco, California, June 5-8, 2006.

6. Dec, John A., Gasbarre, Joseph, and George, Benjamin, E. LT., "Thermal Analysis and Correlation of the Mars Odyssey Spacecrafts' Solar Array During Aerobraking Operations," AIAA 2002 Astrodynamics Conference, Monterey, California, AIAA 2002-4536, August 2002.
7. Dec, John A., and Amundsen, Ruth M., "A Thermal Analysis Approach for the Mars Odyssey

Spacecraft's Solar Array," AIAA 2003 Thermophysics Conference, Orlando, Florida, June 2003.

8. Amundsen, Ruth M., Dec, John A., and George, Ben E.; "Aeroheating Thermal Model Correlation for Mars Global Surveyor (MGS) Solar Array," AIAA Journal of Spacecraft and Rockets, Volume 42, Number 3, May-June 2005, pp 464-473.

9. Carpenter, A. S., "The Magellan Aerobraking Experiment: Attitude Control Simulation and Preliminary Flight Results," AIAA Paper 93-3830, August 1993. 